\title{
Masificación, equidad, educación superior: la universalización de la educación superior cubana*
}

Gerardo Ramos Serpa a Adriana López Falcón ${ }^{b}$

\section{Resumen}

El objetivo del artículo es valorar los debates y retos de la masificación y universalización de la educación superior en varias regiones del mundo y en el caso de Cuba, con la finalidad de apreciar su significado para las políticas públicas y las buenas prácticas que se proponen contribuir mediante la pertinencia y equidad de la educación superior a la sostenibilidad del desarrollo. Se emplean los métodos de análisis documental, analítico-sintético y el enfoque en sistema para, a partir de estudios realizados y documentos sobre esta política pública, caracterizar y apreciar los diferentes puntos de vista y formas de implementación de la masificación de la educación superior en diversos contextos, así como su interdependencia con la equidad social y educativa. Se arriba a evidenciar las divergencias acerca de la masificación de la educación superior, cómo este proceso abarca también la esfera de los estudios de posgrado, y su manifestación en el caso de la universalización de la educación superior cubana.

Palabras clave: Educación superior. Acceso a la educación. Oportunidades educativas. Educación masiva.

\section{Introducción}

El análisis de las tendencias mundiales de la educación superior y sus expresiones regionales y nacionales incluyen en prácticamente todos los casos el reconocimiento del incremento de las personas que hoy acceden a la educación de este nivel.

\footnotetext{
* Esta investigación ha recibido el apoyo de la Universidad Regional Autónoma de Los Andes, Ecuador.

a Universidad Regional Autónoma de Los Andes, Ambato, Ecuador.

b Universidad Regional Autónoma de Los Andes, Ambato, Ecuador.
} 
Así, se considera por la Organización para la Cooperación y el Desarrollo Económicos (OECD por sus siglas en inglés) que:

Durante las últimas décadas, la expansión educativa en todos los países también se ha caracterizado por el considerable crecimiento de la educación terciaria, a menudo considerada como una respuesta a las necesidades de conocimientos de la "economía del conocimiento" para el siglo XXI y a las aspiraciones educativas de las generaciones recientes (OECD, 2017, p. 3).

Al respecto Atria (2012) han reconocido que la expansión de los sistemas de educación superior es probablemente el cambio de mayor alcance y velocidad en el panorama actual del sector y que la literatura suele referirse a esta megatendencia de cambio como la «masificación» de la educación superior.

Junto a ello, las políticas públicas de educación superior deben ir acompañadas del reforzamiento y facilidades que permitan el logro de una mayor equidad en este nivel educacional. Reconociendo a la vez la realización creciente de la tendencia a la masificación de la educación superior a nivel mundial, pero contradictoriamente unida a las disparidades e inequidades en dicho proceso, en el más reciente informe de seguimiento de la educación en el mundo, la Organización de las Naciones Unidas para la Educación, la Ciencia y la Cultura (UNESCO por sus siglas en inglés) ha constatado que la matriculación en este nivel de enseñanza ha continuado incrementándose, llegando en el año 2015 a 213 millones de alumnos; no obstante, también admite que "pocos países garantizan el acceso universal" al mismo (UNESCO, 2017a, p. 20), lo que ha venido acompañado de la transferencia por los gobiernos de sus costos a las familias e individuos (pese a ser reconocido como un derecho y su gratuidad) así como que: "Los más pobres apenas tienen alguna oportunidad de cursar una enseñanza postsecundaria en los países de ingresos bajos y medianos" (UNESCO, 2017a, p. 42).

Unido a esto, en un estudio reciente de la Comisión Económica para América Latina y el Caribe (CEPAL) se valora que las inequidades en la educación superior, en particular en la región latinoamericana, han presentado una tendencia no favorable y que si bien en el período de 1997 al 2013 aunque la cobertura de la educación superior se incrementó del 14 al $21 \%$ de la población, en el quintil de la población de mayores ingresos tal crecimiento fue de un $11 \%$, mientras que en el de ingresos más bajos fue de solo $2 \%$ (CEPAL, 2016). 
Tales retos, que aparecen frente a los procesos de masificación de la educación superior, son enfrentados de variadas formas en diversos países, y en el caso cubano poseen algunas peculiaridades.

A partir de todo ello el objetivo del presente estudio es valorar los debates y retos de la masificación y universalización de la educación superior en varias regiones del mundo y en el caso de Cuba, con la finalidad de apreciar su significado para las políticas públicas y las buenas prácticas que se proponen contribuir mediante la pertinencia y equidad de la educación superior a la sostenibilidad del desarrollo.

\section{Precisiones básicas teórico-metodológicas}

Se emplean los métodos de análisis documental (LÓPEZ-NOGUERO, 2002; PINTO; GÁLVEZ, 1996), analítico-sintético (BEHAR RIVERO, 2008; LOPERA ECHAVARRIA et al., 2010) y el enfoque en sistema (GIGCH, 1990) para, a partir de los estudios realizados y de documentos acerca de esta política pública, analizar, caracterizar y valorar los diferentes puntos de vista, formas de implementación y cuestionamientos acerca de la masificación y universalización de la educación superior en diversos contextos, así como su conexión con la equidad.

En este sentido, se emplearon criterios de búsqueda y selección de materiales, autores y organismos relevantes e ilustrativos de puntos de vista y regiones representativos y de un amplio espectro.

Para ello se comprenden del siguiente modo las principales categorías de análisis aquí empleadas:

- Masificación de la educación superior: expansión cuantitativa del acceso a la educación superior, tanto en los estudios de grado como de posgrado.

- Universalización de la educación superior: fase en el proceso de masificación de la educación superior en la que se alcanzan altos niveles de acceso a dichos estudios.

- Equidad en la educación superior: consideración sin diferenciación de situación socio-económica, política, de género, de etnia, de religión u otras, referida tanto al acceso, la permanencia y el éxito de los estudiantes en el transcurso de los estudios de nivel superior. 


\section{Masificación y equidad en la educación superior}

La masificación de la educación superior se une estrechamente en la visión prospectiva de la UNESCO, a su contribución a la equidad y el desarrollo sostenible $(2014,6)$.

Lo anterior queda explícitamente recogido en los Objetivos de Desarrollo Sostenible de la Organización de las Naciones Unidas (ONU) hasta el año 2030, al considerarse en general en su objetivo 4 la finalidad de garantizar una educación inclusiva, equitativa y de calidad para todos, en tanto su meta 3 plantea para la educación superior que en el 2030 la misma debe asegurar el acceso en condiciones de igualdad para todos los hombres y las mujeres a una formación técnica, profesional y superior de calidad, incluida la enseñanza universitaria (ONU, 2015).

La significación de la masificación de la educación superior se reafirma y completa con el reconocimiento del vínculo entre equidad y calidad en este nivel como elementos indivisibles. Al respecto Aponte-Hernández (2008) considera que la nueva pertinencia social de la educación superior contiene la dimensión de inclusión y equidad que incide y amplía la noción de calidad que prevalece en las instituciones de educación superior.

También es necesario subrayar el vínculo existente entre equidad social y equidad educativa, por el que ambos marchan a la par, se interconectan, interpenetran e influyen recíprocamente.

Así, partiendo de la expansión de la educación superior en los países más desarrollados, expresada por ejemplo en el hecho de que una de cada tres personas adultas posee una calificación de nivel superior aquí, la OECD (2015) reconoce que a la vez las desigualdades tanto en el acceso como en el éxito en la educación superior siguen siendo altas.

De igual modo, la Oficina Regional de la UNESCO para América Latina y el Caribe ha dicho:

El acceso a la educación superior se expandió aceleradamente en la región durante la década del 2000, acumulando un promedio de crecimiento de aproximadamente $40 \%$... Aunque la tendencia a la expansión fue muy extendida, persiste entre los países de la región una enorme heterogeneidad en este nivel educativo. El patrón 
de crecimiento de la educación superior fue, sin embargo, muy inequitativo... (UNESCO, 2013, p. 28).

De aquí que la Conferencia Regional de Educación Superior en América Latina y el Caribe (INSTITUTO DE EDUCACIÓN SUPERIOR PARA AMÉRICA LATINA Y EL CARIBE -IESALC, 2008) haya recomendado que para dar satisfacción al aumento de las exigencias sociales por la educación superior se requiere profundizar en las políticas de equidad para el ingreso e instrumentar nuevos mecanismos de apoyo público a los estudiantes.

Como ejemplo de ello en esta región se puede mencionar que en el caso de la educación superior en Brasil se han dado pasos para atender las cuestiones de ampliar el acceso, la democratización, y la equidad en cuanto a la raza y al género (FELICETTI; CABRERA, 2017; HERNANDES, 2017; POLI, 2018).

Tales procesos se encuentran íntimamente vinculados a la llamada universalización de este nivel educacional.

\section{Universalización de la educación superior}

Asociado al incremento sostenido de las matrículas y al acceso a la educación superior, se ha señalado que un estadío determinado de avance en el proceso de masificación de la educación superior es la llamada universalización de este nivel educacional.

Este término, vinculado al empleo por primera vez por parte de M. Trow en el año 1974 de la idea de un "sistema de educación superior universal", se ha entendido como una fase o grado en el proceso de masificación de la educación superior. Este se ha asociado a diversos indicadores cuantitativos relativos con las personas matriculadas en dicho nivel educacional en relación con el grupo en edad de cursar estos estudios. Así, por ejemplo, el propio Trow (1974) consideraba que ese nivel se alcanzaba a partir del $50 \%$ de personas en la edad correspondiente incorporadas a dicha educación, mientras que Brunner (2005) lo asumía a partir del $75 \%$.

También se emplea el término de post-masificación de la educación superior para referirse a aquellos sistemas de educación superior que han sobrepasado el $50 \%$ de matrícula en este nivel de educación, hablándose de una "era de la post-masificación" de la educación superior a partir de finales de los años $80 \mathrm{del}$ siglo pasado hasta la actualidad. 
Las instituciones de educación superior en la actualidad, y particularmente las universidades, reciben impactos negativos, como la inclinación a la conversión de la educación superior en un negocio que atenta contra la calidad y pertinencia de sus funciones y razón misma de ser. Ello, unido a las tendencias muchas veces contradictorias de masificar el acceso -por un lado-, y la poca permanencia y egreso en este nivel educativo -por otro-, junto a las limitaciones de recursos financieros para el mantenimiento y desarrollo de sus actividades, en su conjunto atenta contra la realización efectiva del carácter verdadero y consecuentemente abierto y equitativo de la educación superior.

\section{Peculiaridades de la masificación y universalización de la educación superior en diversas regiones del mundo}

En este marco, el enfrentamiento a la problemática de la masificación, la equidad y la universalización de la educación superior posee su sello peculiar en diversas regiones del mundo, reconociéndose indistintamente aspectos favorables, cuestionamientos y debates.

Ya en los inicios de este proceso y de su estudio, Trow (1974) señalaba que el crecimiento y la masificación de la educación superior venía acompañado de una variedad de problemas tanto para los sistemas de educación superior que lo experimentaban, como para las sociedades que lo apoyaban. Lo anterior se continúa expresando en momentos más cercanos y en diversas latitudes.

Por ejemplo, en China, según Melo y Zha (2015), este proceso se ha enfrentado a retos tales como la concentración de instituciones de educación superior en áreas urbanas, el financiamiento creciente y diversificado a este proceso combinando fondos generados por las propias universidades y por el gobierno, la diferenciación de los tipos de instituciones proveedoras de educación superior, entre otros.

En la India, Varghese (2015) aprecia como desafíos de la masificación de la educación superior los referidos al aseguramiento de la equidad, la mejora de la calidad, la movilización de fondos que la sustenten, así como la gestión y regulación de dicho proceso.

En África, autores como Mohamedbhai (2014) han valorado que este proceso de masificación de la educación superior se ha llevado a efecto matriculando a 
estudiantes en exceso y por encima de sus capacidades, reflejándose negativamente en la calidad del mismo.

En Europa, estudiosos como Guri-Rosenblit, Sebkova y Teichler (2007) debaten ideas tales como que la diversificación constituye un rasgo común de la ampliación del acceso a la educación superior en la mayoría de los países, pero que ella por sí misma no es suficiente para convertir un sistema de educación superior en orientado a lo masivo.

En los Estados Unidos, su Departamento de Educación reconoció que el acceso a la educación superior norteamericana está excesivamente limitado y que demasiado pocos norteamericanos se preparan, participan y completan la educación superior, especialmente aquellos grupos desatendidos y no tradicionales (UNITED STATES, 2006, p. 8). Recientemente el Subsecretario de Educación de dicho país ha declarado que el acceso siempre será importante, pero solo si los estudiantes que empiezan, terminan (MITCHELL, 2017).

En América Latina, IESALC aprecia que las instituciones de nivel superior deben crecer en diversidad y flexibilidad, sobre todo para garantizar el acceso y permanencia en condiciones equitativas y con calidad para todos, en especial para sectores sociales carentes o vulnerables (2008).

Así mismo, un estudio comparativo de las políticas de masificación de la educación superior en Argentina, Brasil y Chile destaca que más que atender el acceso, es importante dirigir la atención a la permanencia y el éxito en el egreso (VARGAS; HERINGER, 2017).

Como todo lo anterior muestra, la masificación y universalización de la educación superior, estrechamente conectada al tema de la equidad, se encuentra presente en las más variadas latitudes y contextos, acompañada de aciertos, contradicciones y retos.

\section{La universalización de la educación superior en Cuba}

El sistema educacional cubano se compone de los subsistemas de educación preescolar (antes de los 5 años), primaria (1 ro a 6to grado), secundaria ( $7 \mathrm{mo}$ a 9no grado), preuniversitaria (10mo a 12 do grado), técnica-profesional, superior, de adultos y especial, donde la educación primaria y secundaria son obligatorias. 
En relación con el logro de los objetivos de educación para todos establecidos por la UNESCO, pese a que se considera que únicamente uno de cada tres países en el mundo alcanzaron la totalidad de tales metas, se reconoce que en América Latina y el Caribe solo Cuba alcanzó las mismas (UNESCO, 2015).

En este país desde hace más de 58 años se establece y garantiza la naturaleza pública de la educación superior mediante la gratuidad de sus estudios, incluso hasta el nivel de doctorado. Esto, unido a la inversión significativa de fondos en recursos materiales y humanos, con un financiamiento que proviene totalmente del Estado, - expresado en un presupuesto dedicado a la educación superior en el año 2016 que representa más del $2 \%$ del PIB del país (MESA REDONDA, 2016). Vale señalar que los países de la OECD gastan en este nivel educacional como promedio el 1,6\% de su PIB (OECD, 2016).

Para la oferta de plazas de estudio de nivel superior para los estudiantes que culminan su grado 12, se usan los criterios de las demandas que realizan los organismos de la administración central del Estado y los gobiernos locales, de necesidades para su desarrollo económico y social a partir de estudios prospectivos a 5 años; eso, combinado con la posibilidad de ofrecerle la oportunidad de realizar estudios de nivel superior en su propia localidad a aquellas personas que a la vez trabajan, que no pudieron por diversas razones continuar directamente en su momento tales tipos de estudios, o que simplemente lo deseen hacer como parte de sus derechos sociales e intereses individuales.

Ello ha conducido a implementar un proceso conocido como universalización de la educación superior, que ha tenido varias etapas de desarrollo y maduración, comenzando por la campaña de alfabetización en el año 1961 hasta la actualidad. A partir del curso escolar 2001-2002, la universalización de la educación superior en el país recibe un impulso mayor.

Subrayando el vínculo estrecho entre la universalización de la educación superior y la equidad en el caso cubano, el entonces Ministro de Educación Superior expresó que:

el tema de la universalización de la universidad no se aborda de igual forma en todas partes, sobre todo en lo que se refiere a la concepción de su alcance y las formas para lograrlo, pero también sé que es vital para poder concretar una estrategia de desarrollo social que de manera gradual vaya cerrando la brecha entre los hasta ahora siempre 
incluidos y los que cada vez más han ido quedando a la zaga del progreso (VECINO, 2006, p. 2).

Dicho proceso facilita la continuidad de estudios a más amplios sectores de la sociedad, mejora las condiciones de realización de tales estudios al ser los mismos llevados a efecto cerca de los lugares de residencia y trabajo de quienes se incorporan a ello, eleva el nivel de preparación de estas personas incorporadas a la vez a importantes programas de perfeccionamiento de la sociedad cubana y de sus localidades, ocupa el tiempo de estas personas en actividades individual y socialmente útiles, incidiendo así en la disminución de desajustes e inadecuaciones sociales, así como contribuye a la autorrealización personal y al incremento de la autoestima en los mismos.

El modo de implementación de este programa incluye el desarrollo de un modelo pedagógico diseñado especialmente para el mismo, la creación de Filiales y Centros Universitarios Municipales (CUM) que abarcan a todos los municipios del país, la incorporación de profesionales de cada territorio en calidad de profesores a tiempo parcial de las universidades - debidamente categorizados-, la utilización de las instalaciones (escuelas, instituciones culturales, empresas, etc.) y recursos (videos, televisores, computadoras, etc.) disponibles en cada municipio, así como supone un estrecho vínculo entre las autoridades, organizaciones e instituciones de cada territorio.

Un reto que se ha señalado como relevante en el proceso de universalización de la educación superior en Cuba es el de la necesaria atención a la formación pedagógica y didáctica que debe acompañar y garantizarse a los profesores que asumen las labores docentes y formativas en los municipios (ALONSO, 2006).

Algunos datos relevantes acerca de la marcha de este programa de masificación y universalización de la educación superior en Cuba son los siguientes.

Se ofrecen más de 40 carreras universitarias en los municipios en diversas modalidades.

Así mismo, de una matrícula inicial en la educación superior en el curso 2001-2002 de 144553 alumnos (ONE, 2007), se llegó a un máximo de 743979 en el curso 2007-2008, lo que en el curso 2017-2018 se encontró en 224944 matriculados. De dicha matrícula, en este curso el 61,2\% son mujeres (137 687) (ONEI, 2018). 
Gráfico 1. Evolución de la Matrícula Educación Superior Cuba.

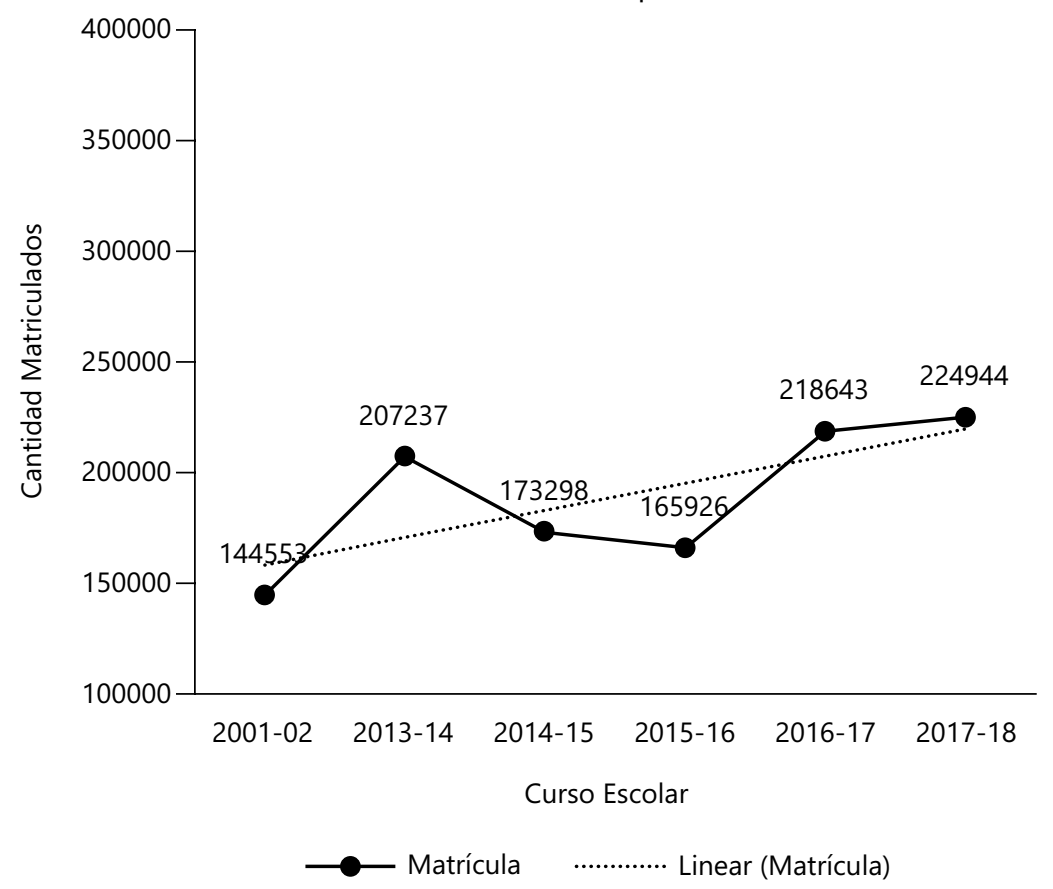

Fuente: ONE (2007, p. 347); ONEI (2018, p. 14).

La evolución de la matrícula en este nivel educacional desde inicios de siglo hasta los últimos 5 años muestra una tendencia ascendente.

En el caso de la matrícula de educación superior en los municipios, de 1142 alumnos en el curso 2001-2002 (CUBA, 2012) se pasó a tener un máximo de 609383 en el curso 2007-2008, lo que en el curso 2017-2018 se encontró en 38 371 matriculados (ONEI, 2018).

La evolución de dicha matrícula en los entornos municipales desde inicios de siglo hasta los últimos 5 años igualmente muestra una tendencia ascendente.

Este proceso también ha estado asociado a la creación de las Filiales Universitarias Municipales, las que en el curso 2006-2007 llegaron a ser 3150 con presencia en los entonces 169 municipios del país. En un periodo más reciente, dichas Filiales se racionalizaron y agruparon en los Centros Universitarios Municipales, los que en el curso 2017-2018 fueron 121 (ONEI, 2018). 
Gráfico 2. Evolución de la Matrícula Educación Superior en los Municipios Cuba.

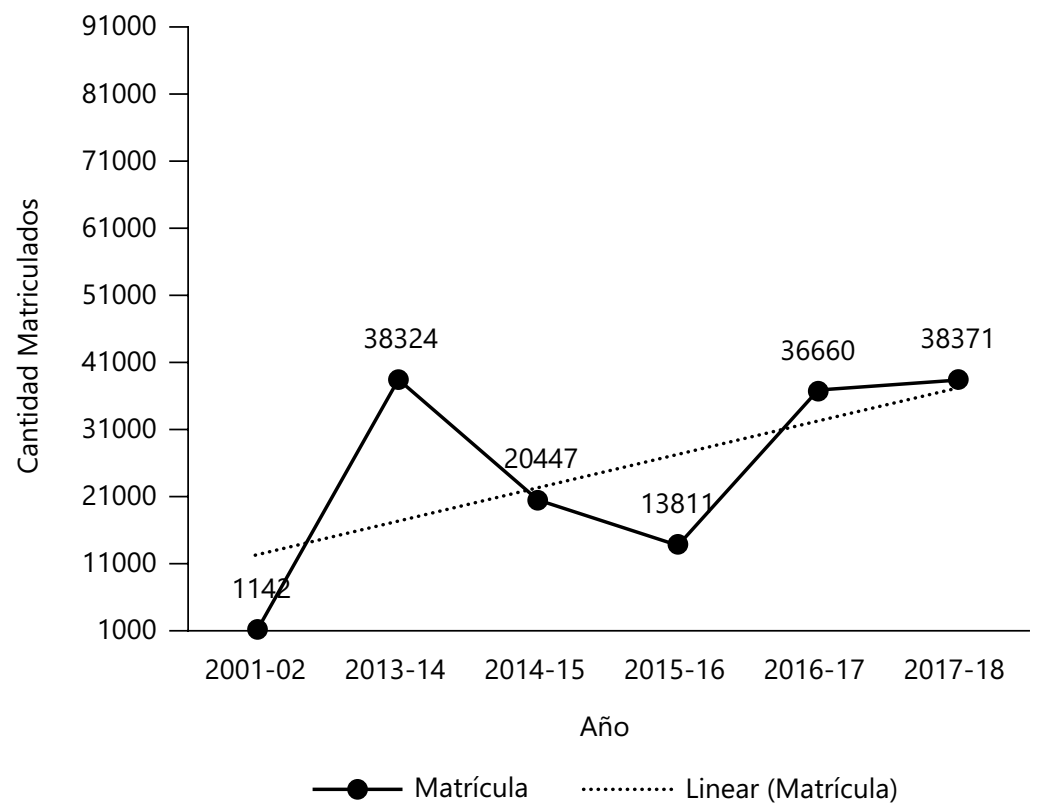

Fuente: CUBA (2012, p. 83); ONEI (2018, p. 14).

Las variaciones de las cifras han estado asociadas a las diferentes etapas del desarrollo y maduración de este proceso de masificación, que ha tenido momentos de crecimiento acelerado, de estabilidad, y de reajuste y racionalidad, en dependencia tanto de las necesidades y demandas, como de las condiciones de satisfacción y aseguramiento.

Aún en momentos de decrecimiento relativo de las matrículas en la educación superior, acompañado de tensiones económicas en el país y el mundo, ello no ha significado una reducción proporcional de los profesores en este nivel de enseñanza. Mientras que en el curso escolar 2001-2002 había 22046 profesores en la educación superior (ONE, 2007), y en el curso 2010-2011 se llegó a un número máximo de 65489 profesores, en el curso 2017-2018 dicha cifra alcanzó los 53313 profesores (ONEI, 2018).

Ello expresa tanto la visión del insustituible papel de los profesores universitarios en el impulso y aporte tanto a la formación de profesionales, como a la investigación y la innovación, unido a la capacitación y superación de los profesionales, técnicos y a la población en general en sus territorios; 
Gráfico 3. Evolución de la Cantidad de Profesores en la Educación Superior Cuba.

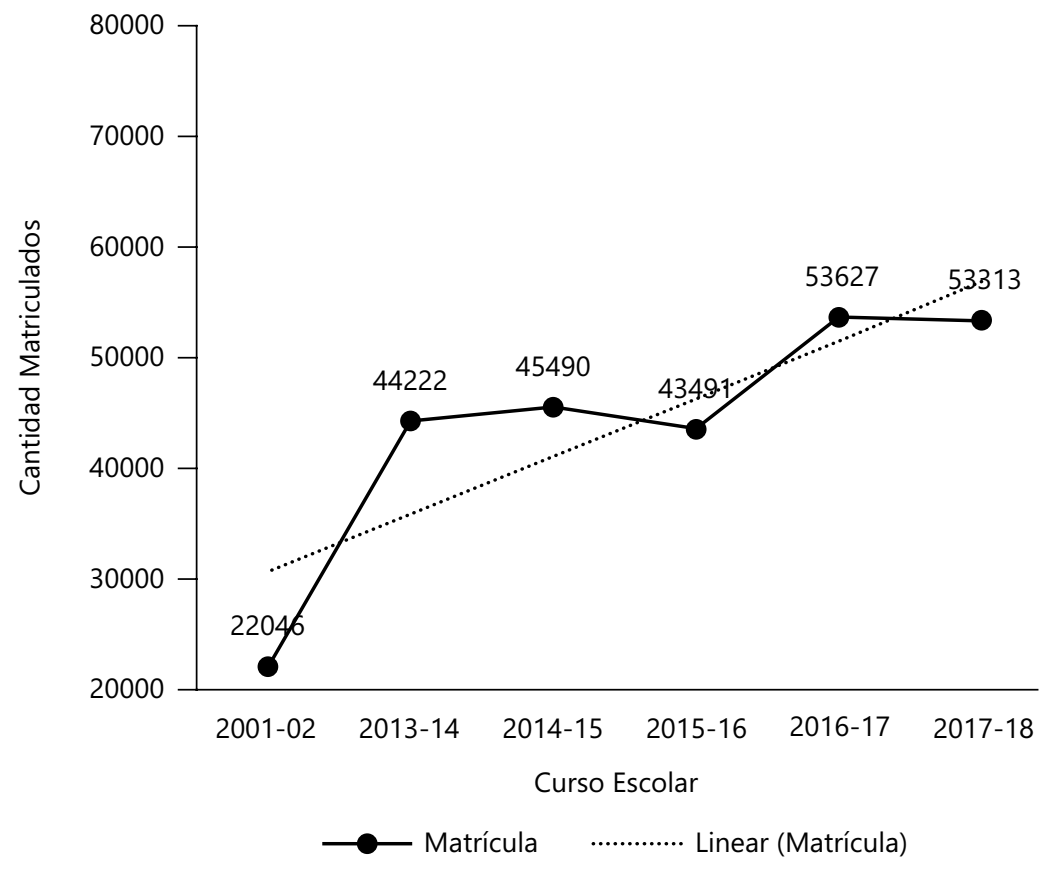

Fuente: ONE (2007, p. 344); ONEI (2018, p. 11).

como la voluntad política del Estado de mantener y apoyar dicha contribución al desarrollo local y del país.

Es de destacar que en un informe relativamente reciente sobre la educación superior en Iberoamérica, se sitúa a Cuba en un nivel de masificación alto (del 55\% o más) de su sistema de educación superior en el curso 2013-2014 (BRUNNER, 2016).

Un elemento que subraya el tema de la equidad en los estudios superiores de este país se refiere a que de los 23478 graduados en este nivel educacional en el curso 2016-2017, el 64,8\% son mujeres (15 235) (ONEI, 2018).

A lo anterior hay que añadir que la universalización de la educación superior cubana abarca también su función de extensión universitaria.

En particular, se ha reconocido y encargado a las universidades y a su presencia en los territorios relevantes e insustituibles, misiones de dinamización y participación en los procesos económicos, productivos y de servicio, de generación de 
conocimiento, tecnología e innovación, socio-comunitarios, de superación de los gobiernos locales y de los actores del desarrollo sostenible en las comunidades, entre otros.

A todo lo expuesto se agrega el proceso de universalización también de los estudios de posgrado.

En el caso de Cuba, un estudio sobre la educación superior en América Latina, destaca que la incorporación al posgrado en el periodo 2000-2005 alcanzó el porcentaje más alto del área, con el 7,7\% de toda la matrícula de nivel superior (IESALC, 2007).

Esta tendencia se reforzó a través de los llamados Programas Académicos de Amplio Acceso (PAAA) que desde hace un tiempo el país ha venido estimulando e implementado como forma de llevar a efecto la universalización de este nivel de enseñanza, con posterioridad llamados Programas de Posgrado en Red (PPR), que permiten realizar estudios de maestría y especialidad de posgrado.

Esta modalidad de posgrado académico se lleva a efecto básicamente en las sedes universitarias municipales. Lo anterior facilita que los profesionales que lo necesiten puedan acceder a estos estudios, y permanecer y egresar de los mismos sin necesidad de desplazarse fuera de los límites de su comunidad, sin abandonar sus responsabilidades laborales ni familiares.

Tales programas se iniciaron en el año 2004 y se han venido desarrollando en diversos campos y modalidades. Los mismos, junto a las formas tradicionales de posgrado, han permitido el tránsito de 126080 estudiantes de posgrado en el año 2001 (ONE, 2007), llegándose a una cifra récord de 656544 participantes en el 2007 (CUBA, 2012), lo que alcanza en el año 2016 la cantidad de 494054 (ONEI, 2018), de los cuales el 59,5\% son mujeres (294 343) (ONEI, 2018), lo cual indica una tendencia creciente en esta esfera.

Se considera que más del $60 \%$ de los graduados universitarios del país cursan tales estudios en la actualidad (BRUNNER, 2016).

En este contexto, es necesario reconocer la estrecha y obligada relación existente entre la universalización de la educación superior y la universalización del posgrado. No se puede ampliar la oferta y el acceso a la formación de profesionales si ello no va acompañado simultáneamente (y quizás mejor con antelación) de la ampliación de los estudios de posgrado, tanto para los 
Gráfico 4. Evolución de la Matrícula de Posgrado Cuba.

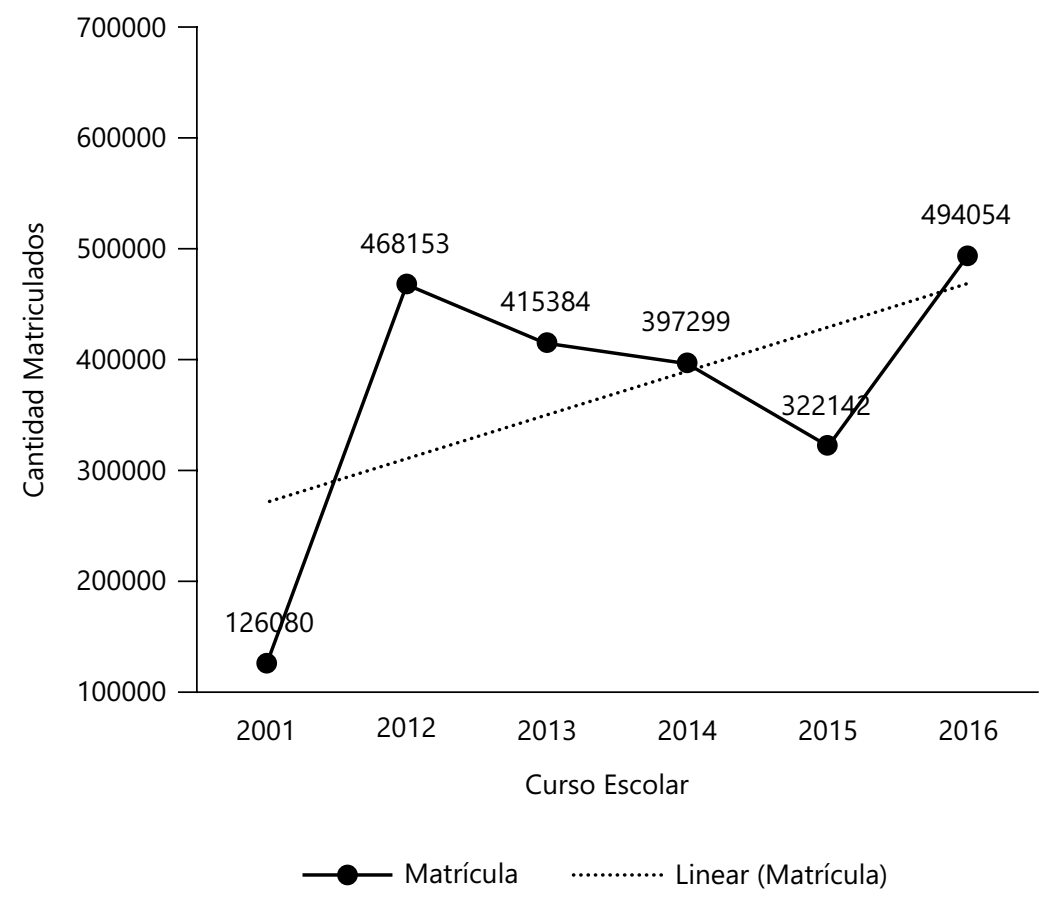

Fuente: ONE (2007, p. 360); ONEI (2018, p. 27).

profesores que atienden dicha masificación como para la formación continua de los egresados.

El proceso de universalización del posgrado en Cuba se encuentra también estrechamente asociado a la premisa de que el posgrado de orientación social debe comprender que las necesidades de producir, distribuir y aplicar conocimientos están subordinadas al desarrollo sostenible, y a la transformación productiva y social con equidad (NÚÑEZ JOVER, 2010).

\section{Debates y retos de la masificación y universalización de la educación superior}

Lo anteriormente expuesto evidencia posiciones divergentes acerca de la masificación de la educación superior, cómo este proceso abarca también la esfera de los estudios de posgrado, y su manifestación en el caso de la universalización de la educación superior cubana, constatándose que tal masificación y su contribución 
efectiva a la equidad es aún insuficiente. Este proceso, a la vez que responde a las políticas y declaraciones de los organismos internacionales sobre el tema, supone una limitada puesta en práctica de las mismas, resultando de utilidad para la apreciación crítica y comprometida en su aplicación.

A la vez, existen posiciones que se contraponen a esta tendencia y su significado real. Así, Hayes y Wynyard (2002) llegan a cuestionar y oponerse abiertamente al proceso de masificación de la educación superior, clasificándolo de "mcdonalización" de este nivel educacional, mientras que Tovar (2012) lo llama "combustible de las máquinas para graduar".

En todo ello influyen las diversas y no siempre acertadas perspectivas teóricas de análisis acerca de este proceso, acompañadas de intereses estrechos o sesgados.

No se puede dejar de notar que el tema de la masificación de la educación superior y la equidad también se conecta con la cuestión de los intereses socioclasistas y de poder en la sociedad. Como fue señalado en la Conferencia Regional de Educación Superior de América Latina y el Caribe: "La inclusión y equidad en la educación superior en la ALyC ha estado vinculada al desarrollo de la estructura de poder e intereses de los grupos dirigentes de las sociedades" (APONTEHERNÁNDEZ, 2008, p. 130).

La universalización de la educación superior tampoco se reduce a una cuestión de oferta de plazas, accesos y algunas facilidades de permanencia.

En el Informe de Seguimiento de la Educación para Todos del año 2008, la UNESCO aconseja que los países no deben centrarse únicamente en la universalización de la enseñanza básica (UNESCO, 2008a) y atender también a este proceso en el resto de los niveles educacionales. Precisamente, en el espíritu de "no dejar a nadie detrás" en educación en general y en particular en la de nivel superior, recientemente la UNESCO ha ofrecido diversas recomendaciones claves para lograr, en el marco de esta tendencia continua y creciente a la masificación de la educación superior a nivel mundial, que la misma sea equitativa y asequible (UNESCO, 2017b).

A tono con ello, y como muestra particular de la cuestión, en correspondencia con las tradiciones, contexto y política social de Cuba, el programa de universalización de la educación superior en dicho país ha permitido no solo hablar de educación para todos, meta ya alcanzada aquí desde la década del 60 del pasado siglo, sino de educación superior para todos, con alta calidad y durante toda la vida. 
Así mismo, en el caso de la masificación de los estudios de posgrado, como lo reconoce un grupo de expertos en este campo: "Como parte del fenómeno de la masificación, cada vez más las calificaciones posgraduadas (maestrías y doctorados) están disponibles ahora en los países industrializados" (UNESCO, 2008b, p. 6), lo que también abarca a otros tipos de países.

Valga aquí como referente general el hecho de que aunque se constata un proceso de masificación del posgrado en relación con el crecimiento de las matrículas del mismo a nivel mundial, ello no significa necesariamente que este se universalice, considerándose que a nivel internacional se ha estado muy lejos de poder hablar de democratización o universalización de los estudios de postgrado, ya que apenas el 1\% de la población puede acceder a los mismos (MORLES, 2006).

La universalización del posgrado a nivel internacional no está exenta de dificultades en cuanto a las posibilidades reales de acceso, tránsito y egreso equitativo y justo por parte de diversos sectores sociales y regiones del mundo. Sobre ello, la directora del Fórum de la UNESCO sobre la Enseñanza Superior, la Investigación y el Conocimiento ha llamado la atención acerca de la necesidad de re-examinar la educación posgraduada en los sistemas de educación superior masificados (KEARNEY, 2010).

Una arista más, asociada a la masificación y universalización de la educación superior, la constituye su vínculo con las posibilidades de empleo. Ante este tema complejo no se debe ser unilateral y vincular única y directamente las motivaciones y sentido de realizar estudios de nivel superior solo a ocuparse y con mayores remuneraciones y ventajas. Este es, sin dudas, un factor relevante. No obstante, el apropiarse de niveles educacionales elevados, incluyendo el posgrado y la superación continua como profesional, también constituye un aspecto de autosatisfacción, aportación al nivel cultural y de mejora personal, junto a su aporte a la integralidad del individuo y a sus aspiraciones de mejora y desarrollo como personalidad, lo que a su vez debe ir acompañado de sus posibilidades de empleo y ejercicio profesional, todo ello visto desde una perspectiva social.

De sobra se conocen los profesionales que no logran ocuparse, al menos en un empleo acorde a sus estudios. Esto sobrepasa los temas educacionales y se enlaza con los de tipo de sistema social y de justicia y ejercicio de los derechos. Valga el comentario de que en Cuba, en el caso de los estudios superiores regulares, junto al título de nivel superior, el Estado es responsable y cumple con la obligación de ofrecer y garantizar una plaza laboral al egresado. Ello se asienta en políticas 
de estudio y demanda anticipada de necesidades de desarrollo sectorial y local para las diferentes profesiones, lo que a su vez no excluye la decisión personal de un individuo (incluso ama de casa) de querer estudiar una carrera y obtener una titulación y superarse por motivaciones personales que no necesariamente se vinculan a querer emplearse o mejorar su estatus laboral, lo que no supone entonces un compromiso estatal de ofrecerle un empleo.

El tema posee otras aristas que no son objeto de análisis en el presente estudio. Solo valga subrayar que se deben estudiar para elevar el desempeño profesional y para realizarse en cuanto a la aportación personal y social de dicho desempeño.

Como acertadamente expresara al respecto el ExSecretario General de las Naciones Unidas Ban Ki-Moon:

Tradicionalmente, las escuelas han preparado a las personas para que aprueben exámenes, pasen al siguiente nivel, se titulen y empiecen a trabajar... No basta con que la educación produzca personas capaces de leer, escribir y contar. La educación debe ser transformadora y dar vida a valores compartidos.... debe también aportar respuestas pertinentes a las grandes cuestiones actuales. Las soluciones tecnológicas, la reglamentación política o los instrumentos financieros no pueden lograr por sí solos un desarrollo sostenible. Se requiere una transformación de nuestra manera de pensar y actuar (2012, p. 21)

Este es un reto no solo para las políticas sociales y los decisores, sino también para cada uno de los ciudadanos en esta compleja época del devenir social.

\section{Consideraciones finales}

La correlación y armonía que debe existir entre masificación, equidad y universalización hace realidad la aspiración de la UNESCO y de lo más genuino del ser humano a que cada vez más amplios sectores de la sociedad tengan acceso a una educación superior no elitista, excluyente ni mercantilizada, a través de la realización efectiva del acceso, permanencia, tránsito, egreso exitoso y educación permanente, permitiendo el libre desarrollo de la inteligencia, el saber, la cultura y la educación permanente frente a los retos de la humanidad en el nuevo milenio. 
La proyección teórica de proponer un enfoque de perspectiva acerca de las llamadas universidades de clase mundial, pero en condiciones de pos-masificación o universalización de la educación superior, muestra la intención de reelaborar y prever la estructura, funciones y tendencias de la educación superior en el futuro frente a sus retos de crecimiento, pertinencia, equidad y calidad.

Se hace cada vez más necesaria entonces una reconceptualización de la comprensión de la universalización de la educación superior como un proceso integral abarcador de todas las funciones sustantivas de este nivel educacional en aras del desarrollo sostenible, la equidad y la realización de todos los ciudadanos.

De igual modo, la universalización de la educación superior puede impactar en la mejora de la sociedad, pero a su vez, es necesario reconocer que ella por sí sola ni de manera automática se refleja en el desarrollo, la equidad, el bienestar de la sociedad.

Claro que no todo lo resuelve la educación. Como ha dicho la UNESCO:

los aumentos de la formación y las competencias no se han traducido siempre por igual en una menor desigualdad social. Junto a los esfuerzos encaminados a ampliar equitativamente la educación, los gobiernos tienen que centrarse en políticas sociales redistributivas para ayudar a invertir la tendencia al ensanchamiento de la desigualdad de ingresos dentro de los países (UNESCO, 2016, p. 16),

considerándose que hacen falta intervenciones sociales y educativas integradas.

Este organismo sostiene que los países más pobres alcanzarán la enseñanza primaria universal más de 100 años después que los más ricos (UNESCO, 2016).

De todo ello, como hipótesis final que ha guiado el trabajo y a la que se puede arribar, se aprecia la alta y trascendente significación de los procesos de masificación y univerzalización de la educación superior tanto para las políticas públicas, como para las buenas prácticas que se proponen contribuir mediante la pertinencia y equidad de la educación superior a la sostenibilidad del desarrollo. Así mismo, se constata que no siempre la comprensión e instrumentación de este proceso se hace con tales fines, sino con interés y perspectivas que deforman la genuina naturaleza de la educación superior y su contribución al desarrollo social y personal; a la vez que existe aún una distancia significativa entre su ideal 
declarado y su aporte efectivo y relevante a la equidad social. En este contexto, los enfoques y la experiencia cubana acerca de dicho proceso pueden ofrecer interesantes y sugestivas aportaciones.

Definitivamente, junto a otros factores y acciones de tipo social y estructural, la universalización de la educación superior, con enfoque realista, con base humanista, con voluntad y financiamiento justo y equitativo comprometido, puede ser un camino aconsejable para avanzar en la equidad social y el desarrollo sostenible efectivo, tan deseado y tan necesario para la humanidad. 


\section{Massificação, equidade, ensino superior: a universalização do ensino superior cubano}

\section{Resumo}

O objetivo do artigo é avaliar os debates e desafios da massificação e universalização da educação superior em várias regiões do mundo e, no caso de Cuba, para apreciar sua relevância para as políticas públicas e as boas práticas que se propõem a contribuir através da pertinência e equidade do ensino superior para a sustentabilidade do desenvolvimento. Os métodos de análise documental, analítico-sintético e abordagem em sistema são utilizados, com base em estudos e documentos sobre esta política pública, para caracterizar e apreciar os diferentes pontos de vista e formas de implementar a massificação da educação superior em diferentes contextos, sua interdependência com a equidade social e educacional. Destacam-se as posições divergentes sobre a massificação do ensino superior, pois esse processo também inclui a esfera dos estudos de pós-graduação e sua manifestação no caso da universalização do ensino superior cubano.

Palavras-chave: Educação superior. Acesso à escola. Oportunidades educacionais. Educação em massa.

\section{Massification, equity, higher education: the universalization of Cuban higher education}

\section{Abstract}

The objective of the article is to assess the debates and challenges of the massification and universalization of higher education in various regions of the world and, in the case of Cuba, in order to appreciate its significance for public policies and good practices that are proposed to contribute through the relevance and equity of higher education to the sustainability of development. The methods of documentary analysis, analytic-synthetic and the system approach are used for, on the basis of studies and documents on this public policy, to characterize and appreciate the different points of view and ways of implementing the massification of higher education in different contexts, their interdependence with social and educational equity. The divergent positions on the massification of higher education are highlighted, how this process also covers the sphere of postgraduate studies and its manifestation in the case of the universalization of Cuban higher education.

Keywords: Higher education. Access to education. Educational opportunities. Mass education. 


\section{Referencias}

ALONSO, M. A. La universalización de la educación superior en Cuba, una experiencia que exige acelerar el proceso de formación pedagógica y didáctica de los profesores en las Sedes Universitarias Municipales. Mendive, Pinar del Río, v. 4, n. 4, p. 230-6, oct./dic. 2006.

APONTE-HERNÁNDEZ, E. Desigualdad, inclusión y equidad en la educación superior en América Latina y el Caribe: tendencias y escenario alternativo en el horizonte 2021. En: GAZZOLA, A. L.; DIDRIKSSON, A. (Eds.). Tendencias de la educación superior en América Latina y el Caribe. Caracas: Iesalc-Unesco, 2008. p. 113-54.

ATRIA, R. Tendencias de la educación superior en América Latina: el contexto para el aseguramiento de la calidad. En: LEMAITRE, M. J.; ZENTENO, M. E. (Eds.). Aseguramiento de la calidad en Iberoamérica: educación superior. Santiago de Chile: Cinda/UE, 2012. p. 163-73. (Informe 2012.).

BEHAR RIVERO, D. S. Metodología de la investigación. Buenos Aires: Shalom, 2008.

BRUNNER, J. J. (Ed.). Educación superior en Iberoamérica. Santiago de Chile: Cinda, 2016. (Informe 2016).

. Tendencias recientes de la educación superior a nivel

internacional: marco para la discusión sobre procesos de aseguramiento de la calidad. Santiago de Chile: Universidad Adolfo Ibáñez, 2005.

COMISIÓN ECONÓMICA PARA AMÉRICA LATINA Y EL CARIBE Cepal. Anuario estadístico de América Latina y el Caribe, 2016. Santiago de Chile, 2017. Disponible en: <http://repositorio.cepal.org/bitstream/ handle/11362/40972/4/S1601037_mu.pdf>. Acceso en: 5 nov. 2017.

CUBA. Ministerio de Educación Superior. Prontuario, curso 2010-2011. Havana, 2012.

FELICETTI, V. L.; CABRERA, A. F. Percurso na educação superior: o ProUni em foco. Ensaio: Avaliação e Políticas Públicas em Educação, Rio de Janeiro, v. 25, n. 95, p. 308-29, 2017. https://doi.org/10.1590/s010440362017002501056

GIGCH, J. P. Teoría general de sistemas. México D. F.: Trillas, 1990. 
GURI-ROSENBLIT, S.; SEBVOKA, H.; TEICHLER, U. Massification and diversity of higher education systems: interplay of complex dimensions. En: GLOBALIZING KNOWLEDGE: EUROPEAN AND NORTH AMERICAN REGIONS AND POLICIES ADDRESSING THE PRIORITY ISSUES OF OTHER UNESCO REGIONS, 2007, Paris. Paris: Unesco, 2007. Disponible en: <http://unesdoc.unesco.org/images/0015/001578/157816e.pdf > . Acceso em: 1 sept. 2017.

HAYES, D.; WYNYARD, R. (Eds.). The mcdonaldization of higher education. Westport: Bergin, 2002.

HERNANDES, P. R. A universidade aberta do Brasil e a democratização do ensino superior público. Ensaio: Avaliação e Políticas Públicas em Educação, Rio de Janeiro, v. 25, n. 95, p. 283-307, 2017. https://doi.org/10.1590/s010440362017002500777

INSTITUTO PARA LA EDUCACIÓN SUPERIOR EN AMÉRICA LATINA Y EL CARIBE - Iesalc. Declaración de la conferencia regional de educación superior en América Latina y el Caribe. Caracas, 2008. Disponible en: $<\mathrm{http} / / /$ www.unesco.org.ve/dmdocuments/declaracioncres_espanol.pdf $>$. Acceso en: 15 oct. 2017.

. Informe sobre la educación superior en América Latina y el Caribe: 2000-2005. Caracas, 2007. Disponible en: <http://www.oei.es/ historico/salactsi/informe_educacion_superiorAL2007.pdf $>$. Acceso en: 2 nov. 2017.

KEARNEY, M. L. Forces shaping postgraduate education: academic credentials in a global context. En: EUROPEAN ASSOCIATION FOR QUALITY ASSURANCE IN HIGHER EDUCATION - ENQA. Quality assurance in postgraduate education. Helsinki: Unesco, 2010. p. 9-14.

KI-MOON, B. Iniciativa mundial: la educación ante todo. París: Unesco, 2012. Disponible en: < https://www.gcedclearinghouse.org/sites/default/files/ resources $/ \% 5 \mathrm{BSPA} \% 5 \mathrm{D} \% 20$ Global\%20Education $\% 20 \mathrm{First} \% 20$ Initiative. pdf $>$. Acceso en: 10 oct. 2018.

LOPERA ECHAVARRÍA, J. D. et al. El método analítico como método natural. Nómadas: Critical Journal of Social and Juridical Sciences, Madrid, n. 25, p. $327-53,2010$.

LÓPEZ-NOGUERO, F. El análisis de contenido como método de investigación. XXI Revista de Educación, Madrid, n. 4, p. 167-79, 2002. 
MELO, D.; ZHA, Q. The massification process in chinese higher education. Revista Gestão Universitária na América Latina, Florianópolis, v. 8, n. 4, p. 134-52, 2015. https://doi.org/10.5007/1983-4535.2015v8n4p134

MESA REDONDA. La universidad cubana mejor preparada en el nuevo curso escolar. Havana, 14 sept. 2016. Disponible en: $<$ http://mesaredonda.cubadebate.cu/mesa-redonda/2016/09/14/la-universidadcubana-mejor-preparada-en-el-nuevo-curso-escolar-video/>. Acceso en: 10 nov. 2017.

MITCHELL, T. Innovating for equity: the future of american higher education. Washington, D.C.: U.S. Department of Education, 2017. Disponible en: $<$ https://www.ed.gov/news/speeches/vision-higher-education-innovation-andopportunity>. Acceso en: 22 oct. 2017.

MOHAMEDBHAI, G. Massification in higher education institutions in Africa: causes, consequences, and responses. International Journal of African Higher Education, Durban, v. 1, n. 1, p. 59-83, 2014. https://doi. org/10.6017/ijahe.v1i1.5644

MORLES, V. Innovaciones relacionadas con educación de postgrado en el siglo XX y perspectivas para el siglo XXI. En: CONGRESO INTERNACIONAL DE EDUCACIÓN SUPERIOR UNIVERSIDAD 2006, 5., 2006, Havana. Memorias... Havana: Ministerio de Educación Superior y las Universidades, 2006. p. 817-24.

NÚÑEZ JOVER, J. Conocimiento académico y sociedad: ensayos sobre política universitaria de investigación y posgrado. Havana: UH, 2010.

\section{ORGANIZACIÓN PARA LA COOPERACIÓN Y EL DESARROLLO} ECONÓMICOS - OECD. Education at a glance 2016: OECD Indicators. Paris, 2016. Disponible en: <http://www.oecd-ilibrary.org/docserver/ download/9616041e.pdf?expires $=1490922681 \& \mathrm{id}=\mathrm{id} \&$ accname $=$ gues t\&checksum $=$ C4C31F79470E61D1765A87044812A2F7>. Acceso en: 14 nov. 2017.

. Educational attainment: a snapshot of 50 years of trends in expanding education. Paris, 2017. Disponible en: $<$ http://www.oecd-ilibrary. org/docserver/download/409ceb2b-en.pdf?expires $=1490030880 \& \mathrm{id}=\mathrm{id} \& a c c n a$ me $=$ guest $\&$ checksum=4D7AAF6915ED18DA4B81DC12861E1E41 $>$. Acceso en: 25 oct. 2017. 


\section{ORGANIZACIÓN PARA LA COOPERACIÓN Y EL DESARROLLO} ECONÓMICOS - OECD. How do differences in social and cultural background influence access to higher education and the completion of studies? Paris, 2015. Disponible en: <http://www.oecd-ilibrary.org/ docserver/download/5jrs703c47s1-en.pdf?expires $=1490030615 \& \mathrm{id}=\mathrm{id} \& \mathrm{a}$ ccname $=$ guest $\&$ checksum $=9063422$ C 85 A5433E801 CE621E0BD18B2 $>$. Acceso en: 17 sept. 2017.

\section{OFICINA NACIONAL DE ESTADÍSTICA - ONE. Anuario} estadistico de Cuba 2006. Havana, 2007. Disponible en: <http:// www.cuba-economia.org/content/download/294/1576/version/1/file/ Anuario+Estad\%C3\%ADstico+2006.pdf>. Acceso en: 16 oct. 2017.

OFICINA NACIONAL DE ESTADÍSTICA E INFORMACIÓN - ONEI. Anuario estadístico de Cuba 2017. Havana, 2018. Disponible en: <http:// www.one.cu/aec2017/18\%20Educacion.pdf $>$. Acceso en: 12 dic. 2018.

\section{ORGANIZACIÓN DE LAS NACIONES UNIDAS - ONU. Transformar} nuestro mundo: la agenda 2030 para el desarrollo sostenible. París, 2015. Disponible en: $<$ http://www.cooperacionespanola.es/sites/default/files/ agenda_2030_desarrollo_sostenible_cooperacion_espanola_12_ago_2015_ es.pdf>. Acceso en: 18 sept. 2017.

\section{ORGANIZACIÓN DE LAS NACIONES UNIDAS PARA LA EDUCACIÓN,} LA CIENCIA Y LA CULTURA - Unesco. Documento de posición sobre la educación después de 2015. París, 2014. Disponible en: <http://unesdoc. unesco.org/images/0022/002273/227336s.pdf > . Acceso en: 2 nov. 2017.

. En América Latina y el Caribe sólo Cuba alcanzó los objetivos globales de la Educación para Todos en el periodo 2000-2015. París, 2015. (Nota de Prensa, 9 abr. 2015). Disponible en: < http://www.unesco.org/new/ es/lima/communities/single-view/news/en_america_latina_y_el_caribe_solo_ cuba_alcanzo_los_objet/>.Acceso en: 21 feb. 2018.

. Informe de seguimiento de la EPT en el Mundo. París, 2008a. Disponible en: $<$ http://unesdoc.unesco.org/images/0015/001591/159125S. pdf $>$. Acceso en: 24 sept. 2017.

. La educación al servicio de los pueblos y el planeta: creación de futuros sostenibles para todos. París, 2016. (Resumen del informe de seguimiento de la educación en el mundo 2016.). Disponible en: $<\mathrm{http} / /$ unesdoc.unesco.org/images/0024/002457/245745s.pdf $>$. Acceso en: 7 oct. 2017. 


\section{ORGANIZACIÓN DE LAS NACIONES UNIDAS PARA LA EDUCACIÓN, LA CIENCIA Y LA CULTURA - Unesco. Rendir} cuentas en el ámbito de la educación: cumplir nuestros compromisos. París, 2017a. (Resumen del informe de seguimiento de la educación en el mundo, 2017/8.). Disponible en: <http://unesdoc.unesco.org/ images/0025/002595/259593s.pdf>. Acceso en: 25 sept. 2017.

. Situación educativa de América Latina y el Caribe: hacia la educación de calidad para todos al 2015. Santiago de Chile: Oficina Regional de Educación para América Latina y el Caribe, 2013. Disponible en: $<\mathrm{http} / /$ www.unesco.org/new/fileadmin/MULTIMEDIA/FIELD/Santiago/images/ SITIED-espanol.pdf $>$. Acceso en: 5 oct. 2017.

\section{. Six ways to ensure higher education leaves no one}

behind. París, 2017b. Disponible en: < http://unesdoc.unesco.org/ images/0024/002478/247862S.pdf>. Acceso en: 11 nov. 2017.

. Trends and issues in postgraduate education: challenges for research. Dublin: Universidad de la Ciudad de Dublin, 2008b. Disponible en: $<$ http://unesdoc.unesco.org/images/0016/001607/160744e.pdf $>$. Acceso en: 1 nov. 2017.

PINTO, M.; GÁLVEZ, C. Análisis documental de contenido: procesamiento de información. Madrid: Síntesis, 1996.

POLI, M. Democratizing the access to college education: brazilian race/color classification in affirmative action's debate. Ensaio: Avaliação e Políticas Públicas em Educação, Rio de Janeiro, v. 26, n. 100, p. 595-618, 2018. https:// doi.org/10.1590/s0104-40362018002601768

TOVAR, E. A. Masificación de la educación superior, combustible de las máquinas para graduar. Educación Siglo XXI 2012, 22 mar. 2012. Disponible en: $<$ http://2012educacion21.blogspot.com/2012/03/masificacion-de-laeducacion-superior.html>. Acceso en: 2 nov. 2017.

TROW, M. Problems in the transition from elite to mass higher education. Berkeley: Carnegie Commission on Higher Education, 1974. Disponible en: $<$ http://files.eric.ed.gov/fulltext/ED091983.pdf>. Acceso en: 25 oct. 2017.

UNITED STATES. Department of Education. A test of leadership: charting the future of U.S. higher education. Washington, D.C., 2006. Disponible en: $<$ http://www.ed.gov/about/bdscomm/list/hiedfuture/reports/pre-pub-report. pdf $>$. Acceso en: 23 oct. 2017. 
VARGAS, H.; HERINGER, R. Políticas de permanência no ensino superior público em perspectiva comparada: Argentina, Brasil e Chile. Archivos Analíticos de Políticas Educativas, Arizona, v. 25, n. 72, p. 1-36, 2017.

VARGHESE, N. V. Challenges of massification of higher education in India. New Delhi: Centre for Policy Research in Higher Education, 2015. Disponible en: < https://www.researchgate.net/profile/NV_Varghese/ publication/292275215_Challenges_of_massification_of_higher_education in_India/links/56ac6fc908ae43a3980a7886.pdf>. Acceso en: 4 nov. 2017.

VECINO, F. La universalización de la universidad por un mundo mejor. En: CONGRESO INTERNACIONAL DE EDUCACIÓN SUPERIOR UNIVERSIDAD 2006, 5., 2006, Havana. Memorias... Havana: Ministerio de Educación Superior y las Universidades, 2006. p. 1-13.

\section{Informações dos autores}

Gerardo Ramos Serpa: Docente de la Universidad Regional Autónoma de Los Andes, Ecuador. Doctor en Ciencias Filosóficas por la Universidad de La Habana en Cuba. Máster en Ciencias de la Educación Superior por la misma Universidad. Licenciado en Filosofía por la Universidad de La Habana. Contato: gramosserpa@gmail.com

iD http://orcid.org/0000-0003-3172-555X

Adriana López Falcón: Docente de la Universidad Regional Autónoma de Los Andes, Ecuador. Máster en Ciencias de la Educación Superior por la Universidad de Matanzas en Cuba. Licenciada en Información Científica y Bibliotecología por la misma Universidad. Contato: alopezfalcon1@gmail.com

iD http://orcid.org/0000-0002-1258-6227 